\title{
Prescription Dose Analysis of Quetiapine in the Elderly and Insomnia
}

\author{
Ki Yong Lee, Seung-Ho Ryu, Jee Hyun Ha, Hong Jun Jeon, and Doo-Heum Park \\ Department of Psychiatry, Konkuk University Medical Center, Seoul, Korea
}

Objective: The purpose of this study is to investigate the appropriate dose of quetiapine in clinical psychiatric diseases by examining the drug prescription dose in the elderly and insomnia group through an analysis of the tendency of quetiapine dose prescribed by psychiatric diagnosis. Methods: Among the patients who had been taking outpatient treatment to the mental health department for about 7 years and 8 months from May 1, 2010 to December 31, 2017, 2,794 patients who were continuously taking quetiapine immediate-release form drugs were retrospectively tested. In addition, all subjects were classified into a total of four groups according to their maintenance dose, four mental diseases that most commonly prescribe quetiapine were selected and grouped, and further analyzed whether there was a difference in prescription capacity by age and comorbidities for the insomnia group. Results: Prescription dose of quetiapine was found to be less than $50 \mathrm{mg}$ in depressive disorders and insomnia, which is a relatively low dose prescribed compared to schizophrenia and bipolar disorder. In the case of insomnia, quetiapine prescribed in the elderly patient group was $30.03 \pm 9.14 \mathrm{mg}$, which was relatively high compared to the nonelderly group. And in the case of insomnia accompanied by depressive disorder, $50.28 \pm 11.41 \mathrm{mg}$ was prescribed, more than $60 \%$ higher doses than that of primary insomnia. Conclusion: In the case of primary insomnia, quetiapine dose prescribed in the elderly patient group is higher than that in the non-elderly patient group.

Keywords: Quetiapine; Elderly; Insomnia

Received: December 6, 2021 Revised: December 13, 2021 Accepted: December 14, 2021

Corresponding author: Doo-Heum Park, MD, PhD, Department of Psychiatry, Konkuk University Medical Center, 120-1 Neungdong-ro, Gwangjin-gu, Seoul 05030, Korea.

Tel: 82-2-2030-7568, Fax: 82-2-2030-7749, E-mail: dhpark@kuh.ac.kr

(a) This is an Open Access article distributed under the terms of the Creative Commons Attribution Non-Commercial License (https://creativecommons.org/licenses/bync/4.0) which permits unrestricted non-commercial use, distribution, and reproduction in any medium, provided the original work is properly cited.

\section{INTRODUCTION}

Quetiapine is one of the most commonly prescribed drugs in the department of psychiatry and used to treat several mental disorders. Quetiapine is a dibenzodiazepine derivative approved by the Food and Drug Administration (FDA) as a treatment of acute mania, depression and schizophrenia $[1,2]$. It is also an offlabel treatment such as anxiety disorder, dementia, autism, obsessive-compulsive disorder, delirium, and insomnia [3].

The Korean Schizophrenia Treatment Guidelines published by the Korean Society of Schizophrenia in 2019 also recommends the treatment of atypical antipsychotics, including quetiapine, for the first stage of antipsychotic treatment for psychotic symptoms [4]. It is prescribed because it is effective in bipolar depression, and treatment effects have also been proven in several clinical trials. Currently, FDA has approved low-dose quetiapine $(300 \mathrm{mg})$ as a treatment for bipolar depression [5]. In the case of depressive disorder, combined treatment of antidepressants and atypical antipsychotics is recommended as an initial treatment strategy if psychotic symptoms are accompanied by major depressive disorders. Quetiapine is also one of the atypical antipsychotic medication recommended for combined treatment [6]. In the case of insomnia, the use of antipsychotics is recommended for insomnia accompanied by psychosis, of which quetiapine and olanzapine are often used as treatments [7].

Quetiapine inhibits histamine 1 (H1) and 5-HT2A receptors, resulting in sedation and anti-anxiety effects [8]. In addition, quetiapine has fewer overall side effects than other antipsychotics, so it is commonly prescribed for the elderly and is often effective without any side effects. Quetiapine is a medication with clear clinical advantages in that it has few side effects and can be used in various doses for various disease spectrum due to its low potency [9]. However, even when used in small doses of $25-200 \mathrm{mg}$, there are side effects such as hepatotoxicity and weight gain, so it 
should be used with caution. In addition, when quetiapine is orally ingested, the clearance rate in the body is reduced by $40 \%$ in the elderly, so the dosage must be adjusted. Therefore, it seems necessary to evaluate the extent to which an appropriate maintenance dose is prescribed by minimizing side effects for the elderly in actual clinical practice.

On the other hand, quetiapine is basically a low-potency drug, meaning that it requires a higher dose to achieve the same effect. In actual clinical practice, it is observed that the dose of quetiapine prescribed for each mental disorder is different, suggesting that the proper maintenance dose and administration period of the same drug may vary depending on the diagnosis. Also, as mentioned above, it is clinically used in low doses for insomnia, and it seems necessary to analyze the current status of prescription for elderly patients. Therefore, the authors decided to classify patients using quetiapine by disease, identify the dosage for each disease, and evaluate the appropriate dose of quetiapine for the elderly and insomnia.

\section{METHODS}

The authors analyzed retrospective medical record data collected from May 2010 to December 2017 of patients who received outpatient treatment in the department of psychiatry. First, each patient's personal information, quetiapine immediate-release form (IR) prescription record, main diagnosis, and sub-diagnosis were investigated through electronic medical records. There were no criteria for selection and exclusion of subjects. This study was conducted with the approval of the Institutional Review Board (IRB approval no. 2020-05-028). Informed consent from the patient was waived by the IRB.

This study first collected basic data from patients who were continuously taking quetiapine IR from May 2010 to December 2017 at the Department of Psychiatry of Konkuk University Medical Center. In addition, a retrospective chart review was performed based on their diagnosis and prescription records. First, according to the maintenance dose, a total of four groups ( $50 \mathrm{mg}$ or less, 75-150 mg, 175-400 mg, more than $400 \mathrm{mg}$ ) were classified. The diagnostic characteristics of each dose group were analyzed. The researchers then selected and grouped the four mental disorders for which quetiapine was most commonly prescribed (depression, bipolar disorder, schizophrenia, and insomnia). Finally, the difference between the average maintenance dose, the maximum and minimum dose, and the dosing period of the quetiapine drug used in each mental disorder was compared.

In addition, in this study, it was additionally investigated whether there was a difference in the dosage of quetiapine prescribed for insomnia according to sex and age. And since it was found that a large number of patients diagnosed with insomnia are accompanied by other psychiatric diseases, the comorbidities were classified into several groups and the average dose of quetiapine prescribed according to age was investigated.

Statistical analysis was performed using PASW 17.0 for WINDOWS (SPSS Inc., Chicago, IL, USA). Multivariate analysis of variance (MANOVA) was performed to simultaneously compare the differences between groups between diseases, and the significance level was set to 0.05 . In addition, classification within insomnia patients was additionally performed, and the Mann-Whitney test was performed to classify differences according to sex and age, and the significance level was set at 0.05 . In addition, the KruskalWallis test was performed to compare the differences between the groups divided by the comorbidities within insomnia, and the significance level was set to 0.01 .

\section{RESULTS}

In Table 1, among a total of 2,794 patients, 1,977 patients are divided into 4 group: schizophrenia, bipolar disorder, depressive disorder, and insomnia. In addition, the patient's demographic data are analyzed according to sex and age, and the prescribed dose and administration period of the drug are analyzed separately. As for the number of patients in the four groups, the depressive disorder group is prescribed quetiapine about six times more than the other group. And in their sex distribution, the male-to-female ratio in the schizophrenia and insomnia group is similar, but in bipolar disorder and depressive disorder, women

Table 1. Demographic data and prescribing quetiapine dose status of the four mental disorder groups $(n=1,977)$

\begin{tabular}{|c|c|c|c|c|}
\hline & $\begin{array}{l}\text { Schizophrenia } \\
\quad(n=262)\end{array}$ & $\begin{array}{l}\text { Bipolar disorder } \\
\quad(\mathrm{n}=244)\end{array}$ & $\begin{array}{l}\text { Depressive disorder } \\
\qquad(\mathrm{n}=1,257)\end{array}$ & $\begin{array}{c}\text { Insomnia } \\
(\mathrm{n}=214)\end{array}$ \\
\hline \multicolumn{5}{|l|}{ Sex } \\
\hline Male & $118(45.0)$ & $81(33.1)$ & $495(39.4)$ & $103(48.3)$ \\
\hline Female & $144(55.0)$ & $163(66.9)$ & $762(60.6)$ & $111(51.7)$ \\
\hline Age (yr) & $49.6 \pm 7.4$ & $49.6 \pm 8.5$ & $57.7 \pm 7.9$ & $65.4 \pm 9.1$ \\
\hline Elderly ${ }^{\dagger}$ & $80(30.5)$ & $71(29.0)$ & $629(50.0)^{*}$ & $141(66.0)^{*}$ \\
\hline \multicolumn{5}{|l|}{ Quetiapine } \\
\hline Maximum dose (mg) & 800 & 800 & 750 & 600 \\
\hline Minimum dose (mg) & 12.5 & 12.5 & 6.25 & 6.25 \\
\hline Average dose (mg) & $230.39 \pm 71.52$ & $188.68 \pm 57.29$ & $81.35 \pm 20.81^{*}$ & $38.93 \pm 13.42$ \\
\hline Average period (months) & $26.17 \pm 8.48$ & $20.54 \pm 4.18$ & $26.85 \pm 5.94$ & $27.85 \pm 9.25$ \\
\hline
\end{tabular}

Data are presented as $\mathrm{n}(\%)$ or mean $\pm \mathrm{SD} .{ }^{*} \mathrm{p}<0.05$; telderly patients are selected based on the age of 60 or older 
in the group accounted for twice as much as men. However, it is not considered as a statistically significant result, and there is no sex difference in the administration of quetiapine. The average age of the group is about 50 years old in the schizophrenia and bipolar disorder group. The average age of the depressive disorder and insomnia group is $57.7 \pm 7.9$ years and $65.4 \pm 9.1$ years, which are higher than that of the schizophrenia and bipolar disorder group. The proportion of elderly patients (over 60 years old) in each group is analyzed. As a result, elderly patients accounted for $50.0 \%(\mathrm{p}=0.04)$ in the depressive disorder group, and $66.0 \%(\mathrm{p}=$ 0.02 ) in the insomnia patient group, and the results are evaluated as statistically significant. This is evaluated as a statistically significant result (Figure 1). As for the maximum and minimum dose by diagnosis, the lowest dose prescribed is $6.25 \mathrm{mg}$ for depression and insomnia, and the maximum dose is $750 \mathrm{mg}$ and $600 \mathrm{mg}$, respectively. When the maximum dose is compared, it can be seen that a lower dose is prescribed for patients with depression and insomnia compared to patients with schizophrenia and bipolar disorder, who are prescribed up to $800 \mathrm{mg}$. The average dose used in patients with schizophrenia is $230.39 \pm 71.52 \mathrm{mg}$, $188.68 \pm 57.29 \mathrm{mg}$ for bipolar disorder, $81.35 \pm 20.81 \mathrm{mg}$ for depres-

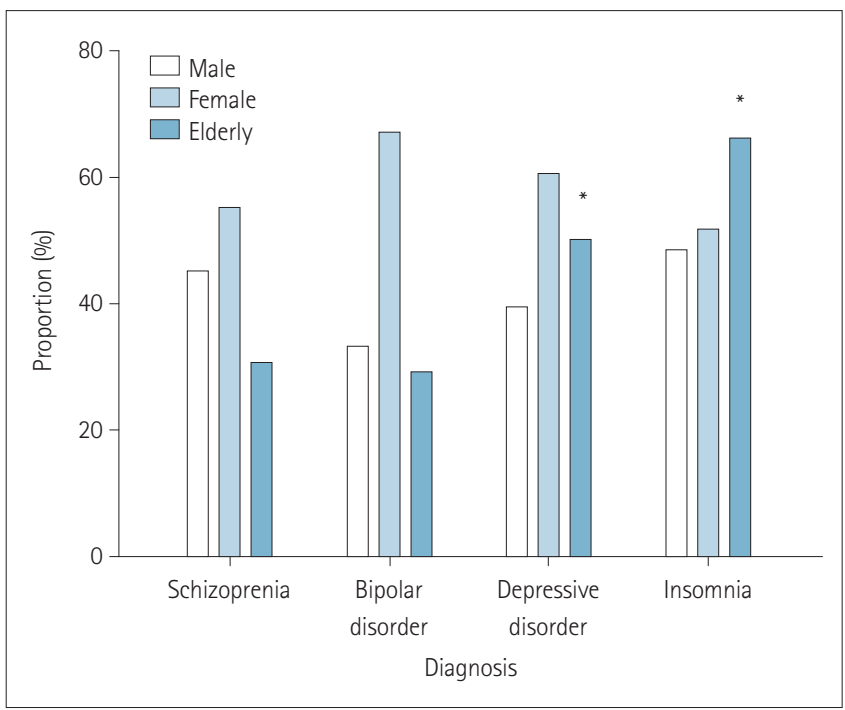

Figure 1. Comparison of the proportions of sex and older adults prescribing quetiapine for each diagnosis. There is no significant difference in the sex ratio by disease $(p>0.05)$. However, a significant increase is observed in the prescription of quetiapine in the elderly group for depressive disorder and insomnia $(p<0.05)$. Elderly patients are selected based on the age of 60 or older. ${ }^{*} p<0.05$. sive disorder, and $38.93 \pm 13.42 \mathrm{mg}$ for insomnia. There is no significant difference in mean dose between patients with schizophrenia and bipolar disorder ( $p>0.05)$, but the difference in that between patients with schizophrenia and bipolar disorder and patients with depressive disorder is evaluated to be significant $(\mathrm{p}=0.01)$. The average prescribing period for quetiapine is $26.17 \pm$ $8.48,20.54 \pm 4.18,26.85 \pm 5.94$, and $27.85 \pm 9.25$ months for schizophrenia, bipolar disorder, depressive disorder, and insomnia, respectively, indicating approximately 2 years of administration.

In Table 2, out of a total of 2,794 patients, schizophrenia, bipolar disorder, depressive disorder, and insomnia are divided into 4 group, and patients who did not belong to these groups are in other disease group. Each group is divided into 4 categories (50 mg or less, 75-150 mg, 175-400 mg, and more than $400 \mathrm{mg}$ ) according to the prescribed quetiapine dose, and the number of patients in each category and the ratio within the diagnosis are calculated and compared. In the schizophrenia group and the bipolar group, it is observed that a relatively high dose of quetiapine of $175 \mathrm{mg}$ or more is prescribed. But it is not evaluated statistically significantly. For depressive disorder and insomnia, the proportion of quetiapine prescribed at $50 \mathrm{mg}$ or less is $70.53 \%(\mathrm{p}=0.01)$ and $91.79 \%(p=0.03)$, respectively. It is evaluated that it occupies a high proportion within each group. It is statistically significant that quetiapine tends to be prescribed in relatively low doses for depressive disorder and insomnia (Figure 2).

Table 3 shows the differences in drug dose by sex and age in the group of patients diagnosed with insomnia. As a result, there is no significant difference in dose according to the sex and age of the group ( $p>0.05)$. There is no difference in quetiapine dose according to age among patients of the same sex. Even in the same age group, the difference in dose according to sex do not show any significance. However, since more than half of the patients diagnosed with insomnia are diagnosed with comorbidity, they are further classified and analyzed.

In Table 4, group of patients diagnosed with insomnia $(\mathrm{n}=214)$ is classified into 5 groups: primary insomnia, depressive disorder, anxiety disorder, psychosis, and other diseases according to comorbidities. Of a total 214 insomnia patients, 101 patients with primary insomnia showed the highest ratio (47.2\%), and 12 patients with insomnia with comorbid mental illness showed the lowest ratio (5.6\%). No significant difference is observed in the comparison of sex or the number of elderly patients for the five classified groups. And in the intergroup comparison of the pre-

Table 2. Comparison of the number of patients according to the prescribed dose of quetiapine classified by diagnosis $(n=2,794)$

\begin{tabular}{|c|c|c|c|c|c|c|}
\hline \multirow{2}{*}{ Diagnosis } & \multicolumn{4}{|c|}{ Quetiapine dose } & \multirow{2}{*}{ Sum } & \multirow{2}{*}{$\mathrm{p}$ value } \\
\hline & $\leq 50 \mathrm{mg}$ & $75-150 \mathrm{mg}$ & $175-400 \mathrm{mg}$ & $>400 \mathrm{mg}$ & & \\
\hline Schizophrenia & $51(19.65)$ & $48(18.49)$ & $86(32.36)$ & 77 (29.47) & 262 & 1.24 \\
\hline Bipolar disorder & $69(28.47)$ & $37(15.23)$ & $77(31.12)$ & $61(25.16)$ & 244 & 0.91 \\
\hline Depressive disorder & $886(70.53)$ & $189(15.04)$ & $117(9.24)$ & $65(5.17)$ & 1,257 & $0.01^{*}$ \\
\hline Insomnia & $196(91.79)$ & $14(6.71)$ & $2(0.75)$ & $2(0.75)$ & 214 & $0.03 *$ \\
\hline Other diseases & $543(66.42)$ & $153(18.78)$ & $88(10.70)$ & $33(4.10)$ & 817 & \\
\hline
\end{tabular}

Data are presented as $\mathrm{n}(\%) .{ }^{*} \mathrm{p}<0.05$ 
scribed average dose of quetiapine, it is found that $30.08 \pm 8.82$ $\mathrm{mg}$ of quetiapine is prescribed for primary insomnia and $51.52 \pm$ $10.12 \mathrm{mg}$ of quetiapine for insomnia accompanied by depressive disorder. This result is evaluated as showing a significant dose difference in which more than $60 \%$ of quetiapine is prescribed for insomnia accompanied by depressive disorder $(p=0.01)$. The average dose for the elderly group with primary insomnia is $30.03 \pm 9.14 \mathrm{mg}$, indicating that the elderly group is prescribed a $20 \%$ higher dose than the non-elderly group $(\mathrm{p}=0.007)$ (Figure 3 ).

\section{DISCUSSION}

Quetiapine has fewer extrapyramidal symptoms and no anti-

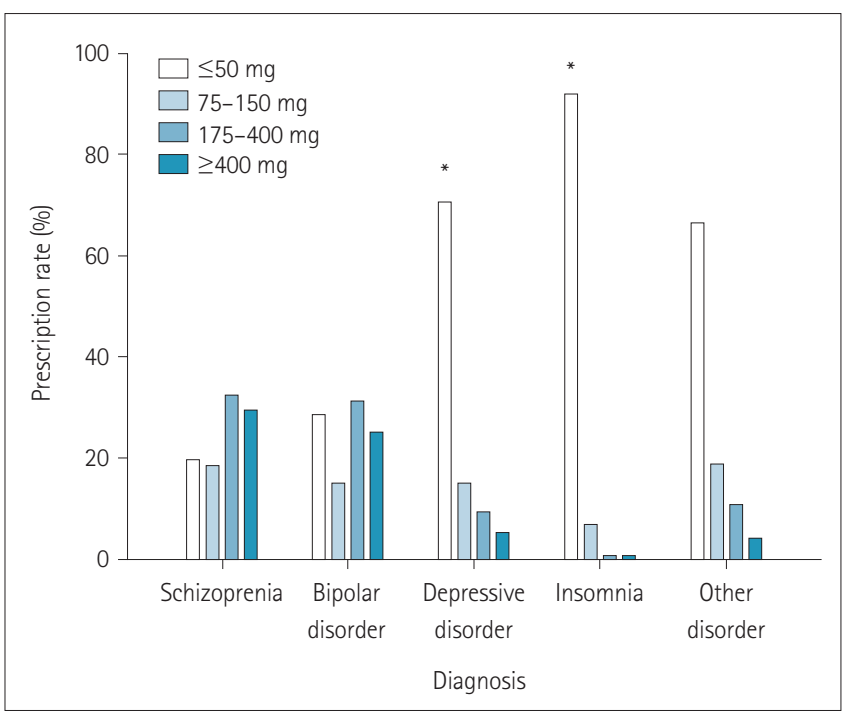

Figure 2. Difference in ratio of quetiapine prescription by dose according to diagnostic characteristics. It is observed that quetiapine is prescribed at a relatively low dose for depression and insomnia compared to other diseases $(p<0.05)$. ${ }^{*} p<0.05$.

Table 3. Current status of quetiapine dose in the insomnia group $(n=214)$

\section{Patients $\quad$ Average dose $(\mathrm{mg})$}

Sex

\begin{tabular}{lrl} 
Male & $93(43)$ & $36.39 \pm 11.22$ \\
Female & $121(57)$ & $40.88 \pm 12.76$ \\
ge & & \\
$<60$ years & $73(35)$ & $39.39 \pm 10.39$ \\
$\geq 60$ years & $141(65)$ & $38.69 \pm 9.52$ \\
\hline
\end{tabular}

Data are presented as $\mathrm{n}(\%)$ or mean \pm SD. cholinergic action compared to other antipsychotics, so it is administered to elderly patients with various neurological diseases for behavioral symptom control [10]. In this study, the proportion of elderly patients who are prescribed quetiapine also showed a significant increase in the insomnia and depressive disorder group compared with other disease groups. The high proportion of the elderly using quetiapine in the insomnia and depression group means that quetiapine is preferred in the elderly. In a recent study, quetiapine is evaluated to show a significantly lower mortality rate, reduced cardiovascular disease risk, and lower metabolic disease compared to other antipsychotics, indicating an advantage for use in elderly patients [11]. However, the risk of falls is evaluated to be high, so it is mentioned that caution should be taken when prescribing for insomnia.

It is mainly studied to be effective in the treatment of primary negative symptoms compared to typical antipsychotics, and to show an effect in combination treatment with clozapine [4]. Although quetiapine is effective in alleviating the positive and negative symptoms of schizophrenia, it may exhibit side effects such as

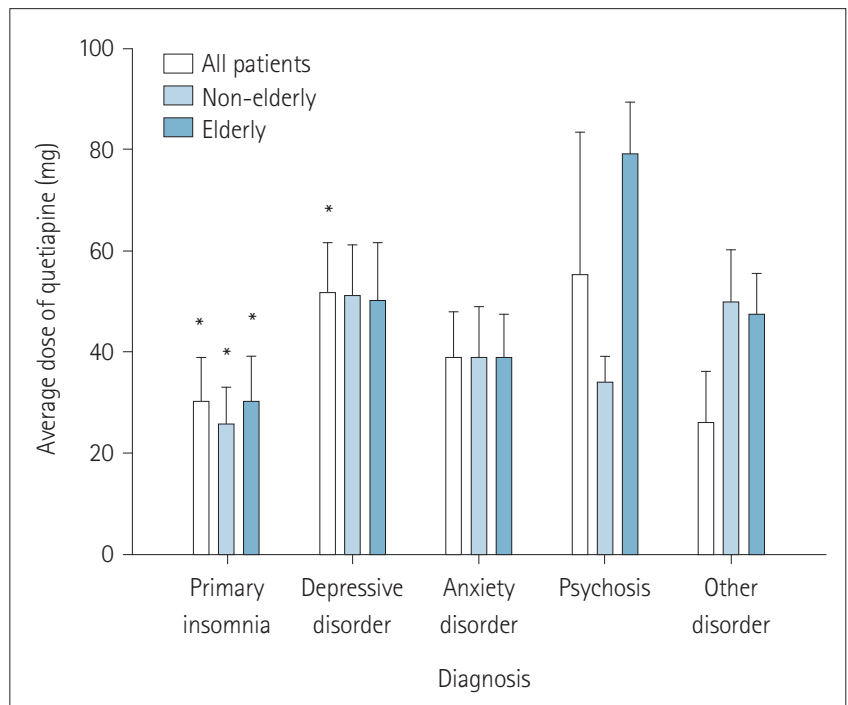

Figure 3. Comparison of average doses for each prescription of quetiapine in elderly and non-elderly patients in the classification of comorbidities of insomnia. A significant increase in the prescribed dose of quetiapine is observed in elderly patients compared to non-elderly patients in the primary insomnia group $(p<0.05)$. It is analyzed that the dose of quetiapine is increased by about $60 \%$ in the insomnia with depressive disorder group compared to primary insomnia group $(p \leq 0.01)$. Elderly patients are selected based on the age of 60 or older. ${ }^{*} \mathrm{p}<0.05$.

Table 4. Current status of quetiapine dose in the insomnia group according to the presence or absence of comorbid diseases $(n=214)$

\begin{tabular}{|c|c|c|c|c|c|c|}
\hline \multirow{2}{*}{ Second diagnosis } & \multirow{2}{*}{ Patients } & \multirow{2}{*}{ Female } & \multirow{2}{*}{ Elderly patient ${ }^{\dagger}$} & \multicolumn{3}{|c|}{ Average dose (mg) } \\
\hline & & & & All & Non-elderly & Elderly $^{\dagger}$ \\
\hline Primary insomnia & $101(47.2)$ & $50(49.0)$ & $80(79)$ & $30.08 \pm 8.82 *$ & $25.52 \pm 7.45^{*}$ & $30.03 \pm 9.14 *$ \\
\hline Depressive disorder & $45(21.0)$ & $31(68.7)$ & $25(55)$ & $51.52 \pm 10.12^{*}$ & $51.25 \pm 9.98$ & $50.28 \pm 11.41$ \\
\hline Anxiety disorder & $32(15.0)$ & $15(47.0)$ & $22(69)$ & $38.65 \pm 9.42$ & $38.75 \pm 10.21$ & $38.54 \pm 8.87$ \\
\hline Psychosis & $12(5.6)$ & $8(66.6)$ & $5(42)$ & $55.20 \pm 28.40$ & $33.92 \pm 5.12$ & $79.17 \pm 10.22$ \\
\hline Other diseases & $24(11.2)$ & $10(42.8)$ & $19(79)$ & $25.93 \pm 10.21$ & $50.00 \pm 10.27$ & $47.50 \pm 8.14$ \\
\hline
\end{tabular}

Data are presented as $\mathrm{n}(\%)$ or mean $\pm \mathrm{SD} .{ }^{*} \mathrm{p}<0.05$; ${ }^{\dagger}$ elderly patients are selected based on the age of 60 or older 
drowsiness (32\%), dizziness (14\%), orthostatic hypotension (13\%), and agitation (11\%). Therefore, the dosage of quetiapine should be prescribed with caution in side effects [11]. Accordingly, in the case of quetiapine IR formulation for the treatment of schizophrenia, quetiapine treatment is usually started at a dose of $50 \mathrm{mg}$, and the maximum prescribed dose can be up to $750 \mathrm{mg}$ by adjusting the quetiapine dose according to the symptoms [12]. In the case of the schizophrenia group in this study, the average dose is $230.39 \pm$ $71.52 \mathrm{mg}$, and the dose is maintained within the FDA-approved dose of 150-750 mg/day. The dose of this quetiapine IR formulation showed the same effectiveness as the standard dose of conventional and other atypical antipsychotic drugs. According to a recent study on the effects of quetiapine on the positive and negative symptoms of schizophrenia, when quetiapine was used for up to 208 weeks and longer, it showed a low incidence of side effects in patients. The average dosing period of quetiapine investigated in this study was 104 weeks, indicating that quetiapine is suitable for a long-term dosing strategy in the treatment of schizophrenia [11].

According to a previous study, it was reported that in the case of bipolar disorder patients with acute manic symptoms, symptoms improved when quetiapine was used more than $600 \mathrm{mg} /$ day. Also, in this study, the maximum dose of $800 \mathrm{mg}$ was administered to the bipolar disorder group showing acute mania [13]. In a recent study, with the use of $400-800 \mathrm{mg} /$ day of quetiapine, the time to relapse is evaluated to increase when taking up to 104 weeks of quetiapine compared to when quetiapine is not used. In addition, in the case of bipolar depressive disorder, when the relapse of depressive episodes significantly loared the reduction in the risk of recurrence of depression compared to placebo when 300 or $600 \mathrm{mg} /$ day is used, it is said that this study suggests the effectiveness of maintaining the medication [14]. Also, in a recent study, a significant decrease in the Montgomery-Åsberg depression rating scale score is reported as a result of $300 \mathrm{mg} / \mathrm{day}$ administration to patients with bipolar depression [5]. In this study, the average maintenance dose of quetiapine in the bipolar disorder group is $188.68 \pm 57.29 \mathrm{mg} /$ day, which is different from the above study results. Therefore, compared with the recent results, the need for additional research through classification within the bipolar disorder group is suggested.

According to a recent study, it is recommended that quetiapine can be added in cases where the initial drug response with antidepressant alone is insufficient even when there are no psychotic symptoms [15]. The FDA approval of quetiapine IR formulation has not been reviewed, but the therapeutic effect is expected through its use as an off-label form in the case of depressive disorder. It is reported that when quetiapine is used alone or in combination with antidepressants, it is effective in the treatment of major depressive disorder [15]. The extended-release formulation is used in combination with antidepressants at a dose of $150-300$ $\mathrm{mg} / \mathrm{day}$, and in this study, the IR formulation is used in combination with an average dose of $81.35 \pm 20.81 \mathrm{mg} /$ day. As for the duration of use, a randomized double-blind trial is conducted for 3 years as a previous study, and the use of paroxetine and quetiap- ine in combination showed significant results on the remission and response rates of depressive symptoms. Therefore, it is suggested that additional investigations are necessary for the results of this study [2].

Benzodiazepines are used for insomnia, but there is a need to be careful about their abuse. Therefore, quetiapine is preferred because it is less likely to be abused and has a sedative effect [16]. Accordingly, it has been reported in previous studies that quetiapine is used as an off-label and is generally used at $25-200 \mathrm{mg} /$ day [17]. This is a dose that takes into account metabolic side effects such as obesity, diabetes, hyperlipidemia, and side effects such as dizziness and dry mouth. A study conducted at $25-75 \mathrm{mg} /$ day for 6 weeks in the treatment of primary insomnia showed significant effects on total sleep time and sleep efficiency as measured by polysomnography and Pittsburgh Sleep Quality Index (PSQI). Therefore, the dose in this study appears to be significant as a therapeutic dose [18]. On the other hand, additional studies on off-label use through the use of quetiapine during insomnia are conducted on insomnia during drug withdrawal and insomnia in Parkinson's disease. It is evaluated that quetiapine 25-100 mg/ day for 6 weeks as a treatment for insomnia of tamoxifen withdrawal symptoms had an immediate effect [19]. Quetiapine was administered at $25-225 \mathrm{mg}$ (average dose $50 \mathrm{mg}$ )/day for up to 60 days for the main symptoms of tamoxifen withdrawal, and it was reported that overall sleep efficiency was improved [20]. When quetiapine is administered at $12.5-50 \mathrm{mg}$ (average dose $31.9 \mathrm{mg}$ )/day for up to 3 months, overall sleep efficiency increased and daytime sleep suppression is achieved for insomnia accompanying Parkinson's disease. The effect of quetiapine on insomnia has been shown to be effective as it is prescribed in low doses for these specific diseases.

When determining the dose of quetiapine, side effects such as prolongation of the QT interval and sedation and cognitive decline in the elderly patient group should be considered. Since there is a side effect of orthostatic hypotension due to its action on adrenergic receptors, quetiapine should be prescribed carefully in the elderly [21]. As a result of examining the effect of quetiapine on insomnia in elderly patients suffering from schizophrenia, it was reported that there was a significant improvement in the PSQI score as a result of administering $50-750 \mathrm{mg} /$ day of quetiapine for 8 weeks. In this study, the elderly patients showed a higher proportion (65\%) than the non-elderly patients in the patients with primary insomnia without comorbidities. When quetiapine was used in a comparative study with risperidone or olanzapine, the incidence of cardiovascular disease was significantly lowered in the elderly [12]. Also, quetiapine showed a lower risk than risperidone in mortality studies [22]. It can be evaluated that quetiapine has a safety advantage compared to other antipsychotics when administered to the elderly. Additional research is needed to determine whether the same effect is shown in primary insomnia.

It is confirmed that in the case of primary insomnia, relatively high doses are prescribed in the elderly than in the young in this study. Quetiapine is used off-label for insomnia among antipsy- 
chotics such as olanzapine and risperidone [23]. This result can be evaluated as meaning that quetiapine is more preferred for off-label use when considering the safety aspect of antipsychotics in elderly patients. Clinically, it has been reported that a large number of doctors are using low-dose quetiapine instead of benzodiazepines to treat insomnia, considering the side effects of benzodiazepines [24]. However, El-Saifi et al. [25] found that the use of quetiapine in the elderly is associated with a higher risk of falls and an increased mortality rate in the Parkinson's population. Therefore, it seems necessary to carefully monitor the use of quetiapine in the elderly.

According to the guidelines from the American Academy of Sleep Medicine, off-label use of antipsychotics is recommended to be avoided. However, due to the well-known sedative effect and insomnia improvement effect at low doses, quetiapine is used off-label for sleep purposes [7]. According to a study on sleep improvement of quetiapine in healthy adult males without comorbidities, it is reported that $25 \mathrm{mg}$ and $100 \mathrm{mg}$ of quetiapine improved sleep quality and efficiency compared to placebo [26]. A study by Atkin et al. [27] conducted in 2018 reported that quetiapine improved sleep for insomnia and had a lower risk of dependence compared to other drugs. In this study, it is confirmed that the low dose of $30.08 \pm 8.82 \mathrm{mg}$ is administered while maintaining the dose for insomnia patients for 27 months.

Quetiapine is generally widely prescribed in patients with other psychiatric comorbidities [24]. In this study, quetiapine is also used in sleep disorders with other comorbidities such as depressive disorder, anxiety disorder, and psychosis. It is reported that $12.5-800 \mathrm{mg}$ of quetiapine IR is prescribed in cases of bipolar disorder, schizophrenia, and comorbidities of depression, improving overall sleep time and enhancing sleep efficiency [28].

It is estimated that $90 \%$ of patients with depression have poor sleep quality [29]. After prescribing quetiapine to insomnia patients suffering from depressive disorder, an improvement in sleep quality was observed as a result of analyzing the change in the PSQI scale [30]. In addition, since quetiapine acts as an antagonist of the $\mathrm{H} 1$ receptor, it is analyzed that quetiapine relieves the symptoms of depression and has a sleep effect in patients with depressive disorder in which the binding of the $\mathrm{H} 1$ receptor is significantly reduced [31]. In this study, quetiapine at a dose of 51.52 \pm $10.12 \mathrm{mg}$ is used as a maintenance dose for the treatment of insomnia in patients with depressive disorder. Quetiapine is prescribed at about $60 \%$ higher dose than that prescribed for primary insomnia.

Several studies have reported that quetiapine has a therapeutic effect on primary insomnia at low doses ( $<100 \mathrm{mg})$. The groups of $25 \mathrm{mg} /$ day and $100 \mathrm{mg}$ /day showed significant improvement in sleep quality compared to placebo, but there is no significant difference between the two groups [26]. Quetiapine is evaluated to be helpful in showing a significant sleep effect even when a relatively low dose is prescribed. However, in the case of insomnia with a comorbid disease, it is analyzed that the effect of sleep appears when quetiapine is prescribed at a relatively high dose. In a study in which about $315 \mathrm{mg}$ of quetiapine was administered for insomnia in patients diagnosed with major depressive disorder for 20 weeks, a significant effect was shown on the Hamilton Rating Scale for Depression insomnia subscale [32]. In the study of quetiapine effect on insomnia in a group of patients with bipolar depression conducted by McElroy et al. [33], a significant improvement in sleep quality is observed at doses of $300 \mathrm{mg}$ and $600 \mathrm{mg}$ compared to placebo. These results suggest that 300-600 mg daily of quetiapine is mainly used for mood disorders, and 25-100 mg daily is recommended for sleep-inducing effects [8]. Higher doses of quetiapine are required for mood disorders compared to primary insomnia at long-term maintenance doses. However, considering that quetiapine exerts an antihistamine effect on the $\mathrm{H} 1$ receptor at a low dose and causes a sedative effect, additional research is needed to determine whether a significant dose difference occurs between the two groups at $100 \mathrm{mg}$ or less.

\section{Limitation}

In this study, it was not possible to evaluate the effectiveness of the prescribed dose because there were no evaluation data to measure how much insomnia symptoms improved in patients receiving quetiapine. Therefore, it could be confirmed that the clinical prescription of quetiapine at a dose similar to that of the previous studies was made clinically, but it was evaluated that it was difficult to confirm that the dose was an appropriate maintenance dose for insomnia.

Also, in the case of the depressed patient group, there were insufficient data to confirm whether quetiapine was prescribed for the treatment of depressive symptoms or was additionally prescribed for the purpose of treating insomnia. Therefore, it was evaluated that there was a limit to making a clear distinction between the insomnia group with depressive symptoms and the depression group.

\section{Conclusion}

Through this study, the authors tried to understand the trends in the prescribed dose of quetiapine according to the diagnostic characteristics and age in psychiatric clinical practice.

Furthermore, it was possible to confirm the difference in the prescribed dose of quetiapine according to each comorbidity within insomnia. Through a retrospective chart review, it was confirmed that quetiapine was being used for various off-label administration as well as for schizophrenia and bipolar disorder. In the group of patients with depressive disorder and insomnia, the proportion of elderly patients was higher than that of other group, and the prescribed dose of quetiapine showed a tendency to be prescribed at a relatively low dose.

In the insomnia group, it was confirmed that the insomnia group with depressive disorder as a comorbidity received a higher dose of quetiapine than the primary insomnia group. And in the case of primary insomnia patients, it was analyzed that the older the patient, the higher the dose was prescribed compared to the nonelderly patient. Through this, we reconfirmed the significance of 
the study results on the effective average dosage in the previous study, and suggested the possibility of using it as a reference data for the treatment of patients in the relevant disease group in the clinical trials.

\section{Acknowledgments}

None

\section{Conflicts of Interest}

The authors have no potential conflicts of interest to disclose.

\section{Author Contributions}

Conceptualization: Ki Yong Lee, Doo-Heum Park. Data curation: Ki Yong Lee, Doo-Heum Park. Formal analysis: Ki Yong Lee, Doo-Heum Park. Funding acquisition: Doo-Heum Park. Investigation: Ki Yong Lee. Methodology: Ki Yong Lee, Doo-Heum Park. Project administration: Ki Yong Lee, Doo-Heum Park. Resources: Ki Yong Lee, Doo-Heum Park. Software: Ki Yong Lee. Supervision: Doo-Heum Park, Seung-Ho Ryu, Jee Hyun Ha, Hong Jun Jeon. Validation: Ki Yong Lee, Doo-Heum Park. Visualization: Ki Yong Lee. Writing_original draft: Ki Yong Lee. Writing_-review \& editing: Doo-Heum Park.

\author{
ORCID iDs \\ Ki Yong Lee (1) \\ https://orcid.org/0000-0001-9611-5889 \\ Seung-Ho Ryu \\ https://orcid.org/0000-0001-8057-8723 \\ Jee Hyun Ha (1) \\ https://orcid.org/0000-0002-0973-1139 \\ Hong Jun Jeon (1) \\ https://orcid.org/0000-0002-0260-0494 \\ Doo-Heum Park \\ https://orcid.org/0000-0002-4833-5366
}

\section{REFERENCES}

1. Bauer M, Pretorius HW, Constant EL, Earley WR, Szamosi J, Brecher M. Extended-release quetiapine as adjunct to an antidepressant in patients with major depressive disorder: results of a randomized, placebo-controlled, double-blind study. J Clin Psychiatry 2009;70:540-549.

2. Hussain MZ, Waheed W, Hussain S, Chaudhry ZA. A comparison of unipolar depression treatment using antidepressants alone versus using antidepressants in combination with quetiapine. Eur Neuropsychopharmacol 2005;15:S453-S454.

3. Philip NS, Mello K, Carpenter LL, Tyrka AR, Price LH. Patterns of quetiapine use in psychiatric inpatients: an examination of off-label use. Ann Clin Psychiatry 2008;20:15-20.

4. Genç Y, Taner E, Candansayar S. Comparison of clozapine-amisulpride and clozapine-quetiapine combinations for patients with schizophrenia who are partially responsive to clozapine: a single-blind randomized study. Adv Ther 2007;24:1-13.

5. Murasaki M, Koyama T, Kanba S, Takeuchi M, Shimizu Y, Arita E, et al. Multicenter, randomized, double-blind, placebo-controlled study of quetiapine extended-release formulation in Japanese patients with bipolar depression. Psychopharmacology (Berl) 2018;235:2859-2869.

6. National Collaborating Centre for Mental Health (UK). Depression: the treatment and management of depression in adults (updated edition). Leicester: British Psychological Society, 2010.
7. Schutte-Rodin S, Broch L, Buysse D, Dorsey C, Sateia M. Clinical guideline for the evaluation and management of chronic insomnia in adults. J Clin Sleep Med 2008;4:487-504.

8. Stahl SM. Dopamine system stabilizers, aripiprazole, and the next generation of antipsychotics, part 1, "Goldilocks" actions at dopamine receptors. J Clin Psychiatry 2001;62:841-842.

9. Goldstein JM. Quetiapine fumarate (Seroquel): a new atypical antipsychotic. Drugs Today (Barc) 1999;35:193-210.

10. Tariot PN, Ismail MS. Use of quetiapine in elderly patients. J Clin Psychiatry 2002;63 Suppl 13:21-26.

11. McManus DQ, Arvanitis LA, Kowalcyk BB. Quetiapine, a novel antipsychotic: experience in elderly patients with psychotic disorders. J Clin Psychiatry 1999;60:292-298.

12. Chatterjee S, Chen H, Johnson ML, Aparasu RR. Comparative risk of cerebrovascular adverse events in community-dwelling older adults using risperidone, olanzapine and quetiapine: a multiple propensity score-adjusted retrospective cohort study. Drugs Aging 2012;29:807-817.

13. Sachs G, Chengappa KN, Suppes T, Mullen JA, Brecher M, Devine NA, et al. Quetiapine with lithium or divalproex for the treatment of bipolar mania: a randomized, double-blind, placebo-controlled study. Bipolar Disord 2004; 6:213-223.

14. Calabrese JR, Keck PE Jr, Macfadden W, Minkwitz M, Ketter TA, Weisler $\mathrm{RH}$, et al. A randomized, double-blind, placebo-controlled trial of quetiapine in the treatment of bipolar I or II depression. Am J Psychiatry 2005;162: 1351-1360.

15. Cutler AJ, Montgomery SA, Feifel D, Lazarus A, Aström M, Brecher M. Extended release quetiapine fumarate monotherapy in major depressive disorder: a placebo- and duloxetine-controlled study. J Clin Psychiatry 2009;70: 526-539.

16. Soldatos CR, Dikeos DG, Whitehead A. Tolerance and rebound insomnia with rapidly eliminated hypnotics: a meta-analysis of sleep laboratory studies. Int Clin Psychopharmacol 1999;14:287-303.

17. Coe HV, Hong IS. Safety of low doses of quetiapine when used for insomnia. Ann Pharmacother 2012;46:718-722.

18. Wiegand MH. Antidepressants for the treatment of insomnia: a suitable approach? Drugs 2008;68:2411-2417.

19. Pasquini M, Speca A, Biondi M. Quetiapine for tamoxifen-induced insomnia in women with breast cancer. Psychosomatics 2009;50:159-161.

20. Terán A, Majadas S, Galan J. Quetiapine in the treatment of sleep disturbances associated with addictive conditions: a retrospective study. Subst Use Misuse 2008;43:2169-2171.

21. Richelson E, Souder T. Binding of antipsychotic drugs to human brain receptors focus on newer generation compounds. Life Sci 2000;68:29-39.

22. Huybrechts KF, Gerhard T, Crystal S, Olfson M, Avorn J, Levin R, et al. Differential risk of death in older residents in nursing homes prescribed specific antipsychotic drugs: population based cohort study. BMJ 2012;344:e977.

23. Anderson SL, Vande Griend JP. Quetiapine for insomnia: a review of the literature. Am J Health Syst Pharm 2014;71:394-402.

24. Kelly M, Dornan T, Pringsheim T. The lesser of two evils: a qualitative study of quetiapine prescribing by family physicians. CMAJ Open 2018;6:E191E196.

25. El-Saifi N, Moyle W, Jones C, Tuffaha H. Quetiapine safety in older adults: a systematic literature review. J Clin Pharm Ther 2016;41:7-18.

26. Cohrs S, Rodenbeck A, Guan Z, Pohlmann K, Jordan W, Meier A, et al. Sleep-promoting properties of quetiapine in healthy subjects. Psychopharmacology (Berl) 2004;174:421-429.

27. Atkin T, Comai S, Gobbi G. Drugs for insomnia beyond benzodiazepines: pharmacology, clinical applications, and discovery. Pharmacol Rev 2018;70: 197-245.

28. Wine JN, Sanda C, Caballero J. Effects of quetiapine on sleep in nonpsychiatric and psychiatric conditions. Ann Pharmacother 2009;43:707-713.

29. Riemann D, Berger M, Voderholzer U. Sleep and depression--results from psychobiological studies: an overview. Biol Psychol 2001;57:67-103.

30. Baune BT, Caliskan S, Todder D. Effects of adjunctive antidepressant therapy with quetiapine on clinical outcome, quality of sleep and daytime motor activity in patients with treatment-resistant depression. Hum Psychopharmacol 2007;22:1-9.

31. Kano M, Fukudo S, Tashiro A, Utsumi A, Tamura D, Itoh M, et al. Decreased histamine $\mathrm{H} 1$ receptor binding in the brain of depressed patients. Eur J Neu- 
rosci 2004;20:803-810

32. Garakani A, Martinez JM, Marcus S, Weaver J, Rickels K, Fava M, et al. A randomized, double-blind, and placebo-controlled trial of quetiapine augmentation of fluoxetine in major depressive disorder. Int Clin Psychopharmacol 2008;23:269-275.
33. McElroy SL, Weisler RH, Chang W, Olausson B, Paulsson B, Brecher M, et al. A double-blind, placebo-controlled study of quetiapine and paroxetine as monotherapy in adults with bipolar depression (EMBOLDEN II). J Clin Psychiatry 2010;71:163-174. 